\title{
Deep Rule-Based Aerial Scene Classifier using High- Level Ensemble Feature Descriptor
}

\author{
Xiaowei $\mathrm{Gu}^{1,2}$ \\ ${ }^{1}$ School of Computing and \\ Communications, \\ ${ }^{2}$ Lancaster Intelligent, Robotic \\ and Autonomous systems (LIRA) \\ Research Centre, \\ Lancaster University \\ Lancaster, UK \\ x.gu3@1ancaster.ac.uk
}

\author{
Plamen P. Angelov ${ }^{1,2}$ \\ ${ }^{1}$ School of Computing and \\ Communications, \\ ${ }^{2}$ Lancaster Intelligent, Robotic \\ and Autonomous systems (LIRA) \\ Research Centre, \\ Lancaster University \\ Lancaster, UK \\ p.angelov@lancaster.ac.uk
}

\begin{abstract}
In this paper, a new deep rule-based approach using high-level ensemble feature descriptor is proposed for aerial scene classification. By creating an ensemble of three pretrained deep convolutional neural networks as the feature descriptor, the proposed approach is able to extract more discriminative representations from the local regions of aerial images. With a set of massively parallel IF...THEN rules built upon the prototypes identified through a self-organizing, nonparametric, transparent and highly human-interpretable learning process, the proposed approach is able to produce the state-of-the-art classification results on the unlabeled images outperforming the alternatives. Numerical examples on benchmark datasets demonstrate the strong performance of the proposed approach.
\end{abstract}

Keywords- deep rule-based, deep convolutional neural network, ensemble feature descriptor, aerial scene classification

\section{INTRODUCTION}

Aerial images are an important source of information for people to understand the Earth [1]. Aerial scene classification is currently a hot research topic because it is instrumental for many real-world applications [2]. Meanwhile, this task is very challenging due to the highly complex semantic contents and spatial patterns of such images.

There have been many approaches proposed for classifying the aerial images. In general, they can be divided into three main categories [3]: 1) low-level methods; 2) middle-level methods; and 3) high-level methods.

Low-level methods attempt to distinguish aerial scenes based on the low-level visual features extracted from the images [4]-[6]. Middle-level methods, in general, encode the low-level visual features extracted from the local regions of the aerial images into holistic middle-level representations for scene classification [7], [8]. High-level methods are, mostly, based on deep convolutional neural networks (DCNNs) [2], [9], [10]. In comparison to the former two categories, highlevel methods can perform classification with the highest accuracy and are the state-of-the-art in the remote sensing domain. Nonetheless, practically all of the existing high-level methods lack transparency in the approximate reasoning process, and the reasons for making a particular decision are often not interpretable for humans [11]. These demerits largely influence the applicability of the high-level approaches in realworld scenarios.

As a recently introduced generic approach for image classification, deep rule-based (DRB) classifier [12], [13] is a powerful alterative to the DCNN models. The DRB approach expands the traditional fuzzy rule-based (FRB) systems with a massively parallel multi-layer structure that DCNNs benefit from [13]. Thanks to the prototype-based nature, the system structure of the DRB approach is fully transparent, and the learning and decision-making processes are autonomous, nonparametric and highly interpretable for humans [11].

The DRB classifier may employ different types of descriptors for visual feature extraction, which can be lowlevel [14], [15], middle-level [8] or high-level [16]-[18]. In reality, however, using one type of feature is often not enough for classifying different scene categories that share similar appearances. Different feature descriptors have different merits and demerits, and they have different descriptive abilities. Therefore, fusing multiple features into more descriptive representations usually results in a stronger classification performance [1], [19], [20].

Following this principle, in this paper, a deep rule-based (DRB) approach using multiple features is proposed for aerial scene classification. The proposed approach uses an ensemble of different high-level feature descriptors for extracting highly distinctive semantic representations from sub-regions of aerial images locally. The DRB system identifies a number of prototypes during the training process, and self-organizes a set of massively parallel prototype-based IF...THEN rules for classification in an autonomous, nonparametric manner [11][13]. Numerical examples demonstrate that the proposed DRB approach is able to produce the state-of-the-art classification results outperforming the alternatives by incorporating the high-level ensemble feature descriptor.

\section{ThE EMPLOYED HIGH-LEVEL FEATURE DESCRIPTORS}

In this section, we briefly introduce the three pre-trained DCNNs that will be used as the high-level feature descriptors in the proposed approach. It has to be stressed that the pretrained DCNN models are used directly without tuning. 


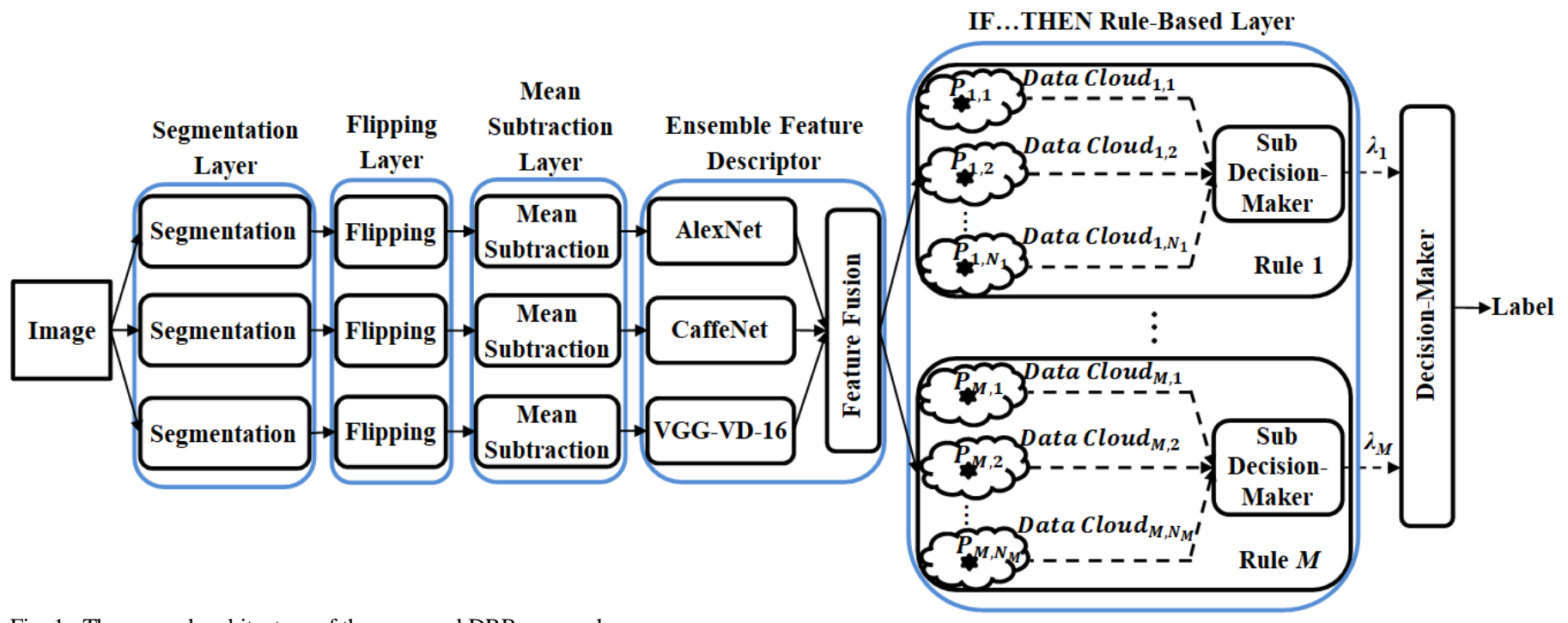

Fig. 1. The general architecture of the proposed DRB approach

\section{A. AlexNet}

AlexNet [18] was the winning DCNN model of the ImageNet Large Scale Visual Recognition Challenge (ILSVRC) in 2012. It largely popularized the applications of DCNNs and has become a baseline model of DCNNs. AlexNet was trained on ImageNet dataset, which contains 1.3 million images. It has eight layers with weights. The first five are convolutional layers, the first and second of which are followed by the normalization layers. The two normalization layers and the fifth convolutional layer are also followed by one max-pooling layer, respectively. The remaining three layers with weights are fully-connected [18]. The last fullyconnected layer is linked to a softmax layer which produces the output of the network. To reduce overfitting, AlexNet involves data augmentation by cropping smaller-size segments and horizontally flipping these segments from original images. This operation artificially creates more training images. The size of input images required by AlexNet is $227 \times 227$ pixels. In this paper, we use the $1 \times 4096$ dimensional activations from the first fully connected layer as the feature vectors of the input images.

\section{B. CaffeNet}

CaffeNet [16] has a similar architecture to AlexNet and is also trained on the image set of ILSVRC 2012. Nonetheless, there are two differences between CaffeNet and AlexNet [3]: 1) the order of max-pooling and normalization layers are exchanged; 2) data augmentation is not used. The size of input images required by CaffeNet is $227 \times 227$ pixels. Similarly, the $1 \times 4096$ dimensional activations from the first fully connected layer are used as the feature vector of the input images [2].

\section{VGG-VD-16 Model}

VGG-VD-16 was introduced in [17] and is one of the bestperforming pre-trained DCNN models. This model has 16 layers with weights. The first 13 layers are convolutional and the last three are fully-connected. The second, fourth, seventh, tenth, thirteenth convolutional layers are followed by a maxpooling layer. There is no normalization layer used in the VGG-VD-16 model. This model is also trained on the image set of ILSVRC 2012 [17]. VGG-VD-16 model requires the input images with the size of $224 \times 224$ pixels. We also extract the $1 \times 4096$ dimensional activations from the first fully connected layer as the feature vectors of the input images [2].

It has to be stressed that, practically, one can use any types of high-level feature descriptors, i.e. GoogLeNet [21], ResNet [22], PlacesNet [23], etc., and any number of them to create an ensemble for feature extraction. The main purpose of this paper, however, is to introduce the general concept and principles of the DRB classifier using ensemble feature descriptor. Therefore, we only use the most representative three DCNNs to create the ensemble descriptor. Nonetheless, one can try different combinations as well.

\section{The PROPOSED DEEP RULE-BASED APPROACH}

The general architecture of the proposed DRB approach is depicted in Fig. 1. As one can see that, the DRB classifier is composed of the following layers:

1) Segmentation layer;

2) Flipping layer;

3) Mean subtraction layer;

4) Ensemble feature descriptor layer;

5) IF...THEN rule-based layer;

6) Decision-maker.

\section{A. Segmentation Layer}

This layer crops five sub-images of the required sizes ( $224 \times 224$ or $227 \times 227$ pixels depending on the DCNN models connected) from the central area and four corners of each image for data augmentation [2], [18]. This type of segmentation enables the DRB classifier to grasp more local 
semantic information from the images and improve the generalization ability.

\section{B. Flipping Layer}

This layer flips each segment of the images horizontally, vertically, and in both direction. The flipping operation further creates three new segments from each segment [2], [18].

The segmentation and flipping layers, in total, produce $K_{o}=20$ segments from each input image, which will be used for feature extraction. An example of the data augmentation is given in Fig. 2 for illustration.

\section{Mean Subtraction Layer}

This layer subtracts from each segment its mean, and centralizes the three channels of the segment around the zero mean. This pre-processing technique helps the pre-trained DCNN models to perform faster because gradients act uniformly for each channel.

\section{Ensemble Feature Descriptor Layer}

The ensemble feature descriptor, as described in Section II, consists of three different pre-trained DCNN models: 1) AlexNet, 2) CaffeNet and 3) VGG-VD-16, and a feature fusion sub-layer.

For each segment, denoted by $\mathbf{s}$, each model will produce a $1 \times 4096$ dimensional feature vector from the activations of the first fully connected layer. The feature fusion sublayer will combine the three feature vectors into a $1 \times 12288$ dimensional semantic representation by the following equation:

$$
x \leftarrow\left[\frac{\mathbf{A N}(\mathbf{s})}{\|\mathbf{A N}(\mathbf{s})\|}, \frac{\mathbf{C N}(\mathbf{s})}{\|\mathbf{C N}(\mathbf{s})\|}, \frac{\mathbf{V V}(\mathbf{s})}{\|\mathbf{V V}(\mathbf{s})\|}\right]^{T}
$$

where $\mathbf{A N}(\mathbf{s}), \mathbf{C N}(\mathbf{s}), \mathbf{V V}(\mathbf{s})$ represent the feature vectors extracted by AlexNet, CaffeNet and VGG-VD-16 models, respectively. In equation (1), $\mathrm{L}_{2}$-normalization is used to guarantee that the feature vectors from the three models contribute equally in the semantic representation.

\section{E. IF ...THEN Rule-Based Layer}

The IF...THEN rule base in this layer is the "core" of the DRB classifier. Assuming the datasets has $M$ categories, the DRB classifier will self-organize and self-update $M$ massively parallel IF...THEN rules from prototypes that are identified from the segments of the images of each category (one rule per category) in a nonparametric, transparent and human-interpretable manner. Each IF...THEN rule has the following form $(m=1,2, \ldots, M)[11]-[13]$ :

$$
\begin{gathered}
I F\left(\mathbf{s} \sim \mathbf{P}_{m, 1}\right) \operatorname{OR}\left(\mathbf{s} \sim \mathbf{P}_{m, 2}\right) \text { OR } \ldots \text { OR }\left(\mathbf{s} \sim \mathbf{P}_{m, N_{m}}\right) \\
\text { THEN }(\text { category } m)
\end{gathered}
$$
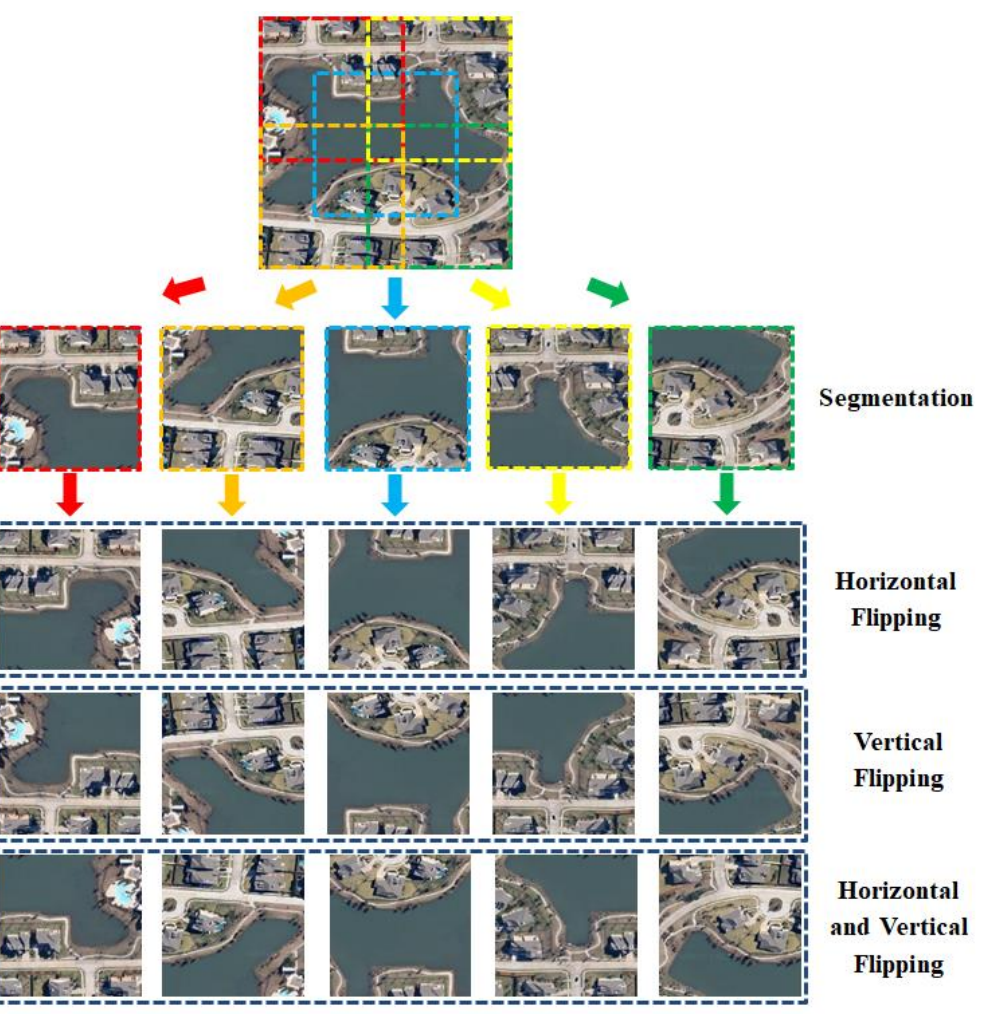

g. 2. Illustrative example of data augmentation

where " " denotes similarity, which can be seen as a fuzzy degree of membership; $\mathbf{s}$ is a particular segment with $\boldsymbol{x}$ as its feature vector; $\mathbf{P}_{m, i}$ denotes the $i^{\text {th }}$ prototype of the $m^{\text {th }}$ category with $\boldsymbol{p}_{m, i}$ as the corresponding feature vector; $N_{m}$ is the number of identified prototypes of the $m^{\text {th }}$ category.

Each IF...THEN rule contains a number of prototypes that are connected by a sub-decision maker using the "winnertakes-all" principle. Therefore, each IF...THEN rule is a massively parallel series of singleton fuzzy rules of AnYa type [24] connected by logical "OR" operators .

The detailed identification process of the IF...THEN rules have been given in [11], [13]. One can also download from [25] the open source software implemented in Matlab with detailed instructions provided in [11]. To make this paper selfcontained, the identification process of the rule base is summarized by the following pseudo-code. It has to be noticed that because the IF...THEN rules are identified in parallel, we present the identification process of the $m^{\text {th }}$ rule as an example. The same principles can be applied to the other IF...THEN rules within the same rule base.

\begin{tabular}{|l|}
\hline INPUT: streaming images of the $m^{\text {th }}$ category \\
ALGORITHM BEGINS \\
\hline While a new segment, $\mathbf{s}_{m, k}$ is available: \\
i. Extract the semantic representation, $\boldsymbol{x}_{m, k}$ from $\mathbf{s}_{m, k} ;$ \\
ii. Normalize $\boldsymbol{x}_{m, k}$ by its $\mathrm{L}_{2}$ norm:
\end{tabular}




$$
\boldsymbol{x}_{m, k} \leftarrow \frac{\boldsymbol{x}_{m, k}}{\left\|\boldsymbol{x}_{m, k}\right\|}
$$

iii. If $(k=1)$ Then

1. Initialize the global meta-parameters:

$N_{m} \leftarrow 1 ; \quad \boldsymbol{\mu}_{m} \leftarrow \boldsymbol{x}_{m, k}$

where, $N_{m}$ is the number of prototypes; $\boldsymbol{\mu}_{m}$ is the global mean of the feature vectors of the images of the $m^{\text {th }}$ category.

2. Initialize the meta-parameters of the first data cloud, $\mathbf{C}_{m, N_{m}}$ :

$\mathbf{C}_{m, N_{m}} \leftarrow\left\{\mathbf{s}_{m, k}\right\}$

$\mathbf{P}_{m, N_{m}} \leftarrow \mathbf{s}_{m, k}$

$\boldsymbol{p}_{m, N_{m}} \leftarrow \boldsymbol{x}_{m, k}$

$S_{m, N_{m}} \leftarrow 1$

$r_{m, N_{m}} \leftarrow r_{o}$

where $S_{m, N_{m}}$ is the support (number of members) of $\mathbf{C}_{m, N_{m}} ; r_{m, N_{m}}$ is the corresponding radius of influential area; $r_{o}$ is a constant to stabilize the new data cloud and $r_{o}=\sqrt{2\left(1-\cos \left(30^{\circ}\right)\right)}$ [11], [13].

3. Initialize the IF...THEN rule:

$$
\mathbf{R}_{m}: \operatorname{IF}\left(\mathbf{s} \sim \mathbf{P}_{m, N_{m}}\right) \quad \operatorname{THEN}(\text { category } m)
$$

$i v$. Else

1. Update global mean:

$$
\boldsymbol{\mu}_{m} \leftarrow \frac{k-1}{k} \boldsymbol{\mu}_{m}+\frac{1}{k} \boldsymbol{x}_{m, k}
$$

2. Calculate the data density at $\mathbf{s}_{m, k}$ and prototypes $\mathbf{P}_{m, j}$

$$
\left(j=1, \ldots, N_{m}\right) \text { [26], [27]: }
$$

$$
D(\mathbf{z})=\frac{1}{1+\frac{\left\|z-\boldsymbol{\mu}_{m}\right\|^{2}}{1-\|z\|^{2}}}
$$

where $\mathbf{z}=\mathbf{s}_{m, k}, \mathbf{P}_{m, 1}, \mathbf{P}_{m, 2}, \ldots, \mathbf{P}_{m, N_{m}}$;

$$
\boldsymbol{z}=\boldsymbol{x}_{m, k}, \boldsymbol{p}_{m, 1}, \boldsymbol{p}_{m, 2}, \ldots, \boldsymbol{p}_{m, N_{m}} .
$$

3. Find the nearest data cloud, $\mathbf{C}_{m, n^{*}}$ :

$$
n^{*} \leftarrow \underset{j=1,2, \ldots, N_{m}}{\arg \min }\left(\left\|\boldsymbol{x}_{m, k}-\boldsymbol{p}_{m, j}\right\|\right)
$$

4. If $\left(D\left(\mathbf{s}_{m, k}\right)>\max _{j=1,2, \ldots, N_{m}}\left(D\left(\mathbf{P}_{m, j}\right)\right)\right)$

Or $\left(D\left(\mathbf{s}_{m, k}\right)<\min _{j=1,2, \ldots, N_{m}}\left(D\left(\mathbf{P}_{m, j}\right)\right)\right)$

Or $\left(\left\|\boldsymbol{x}_{m, k}-\boldsymbol{p}_{m, n^{*}}\right\|>r_{m, n^{*}}\right)$ Then:

- Add a new data cloud:

$N_{m} \leftarrow N_{m}+1$

$\mathbf{C}_{m, N_{m}} \leftarrow\left\{\mathbf{s}_{m, k}\right\}$

$\mathbf{P}_{m, N_{m}} \leftarrow \mathbf{s}_{m, k}$

$$
\begin{aligned}
& \boldsymbol{p}_{m, N_{m}} \leftarrow \boldsymbol{x}_{m, k} \\
& S_{m, N_{m}} \leftarrow 1 \\
& r_{m, N_{m}} \leftarrow r_{o}
\end{aligned}
$$

5. Else:

- Update the meta-parameters of $\mathbf{C}_{m, n^{*}}$ :

$$
\begin{aligned}
\mathbf{C}_{m, n^{*}} & \leftarrow \mathbf{C}_{m, n^{*}}+\left\{\mathbf{s}_{m, k}\right\} \\
\boldsymbol{p}_{m, n^{*}} & \leftarrow \frac{S_{m, n^{*}}}{S_{m, n^{*}}+1} \boldsymbol{p}_{m, n^{*}}+\frac{1}{S_{m, n^{*}}+1} \boldsymbol{x}_{m, k} \\
S_{m, n^{*}} & \leftarrow S_{m, n^{*}}+1 \\
r_{m, n^{*}} & \leftarrow \sqrt{\frac{r_{m, n^{*}}^{2}+\left(1-\left\|\boldsymbol{p}_{m, n^{*}}\right\|^{2}\right)}{2}}
\end{aligned}
$$

6. End If

7. Update the IF...THEN rule:

$$
\mathbf{R}_{m}: \begin{gathered}
I F\left(\mathbf{s} \sim \mathbf{P}_{m, 1}\right) \text { OR ... OR }\left(\mathbf{s} \sim \mathbf{P}_{m, N_{m}}\right) \\
\operatorname{THEN}(\text { category } m)
\end{gathered}
$$

\section{$v$. End If}

End While

\section{ALGORITHM ENDS}

OUTPUT: the massively parallel IF...THEN rule $\mathbf{R}_{m}$

\section{F. Decision-Maker}

During the validation process, for a particular segment, $\mathbf{s}_{i}$ $(i=1,2, \ldots, 20)$ from an unlabelled image, $\mathbf{I}$, one can obtain $M$ scores of confidence with the corresponding $M$ massively parallel IF...THEN rules (one score per rule). Each score of confidence is formulated as follows $(m=1,2, \ldots, M)$ :

$$
\lambda_{m}\left(\mathbf{s}_{i}\right)=\max _{j=1,2, \ldots, N_{m}}\left(e^{-\left\|\boldsymbol{x}_{i}-\boldsymbol{p}_{m, j}\right\|^{2}}\right)
$$

where $x_{i}$ is the corresponding $12288 \times 1$ dimensional semantic representation of $\mathbf{s}_{i}$.

The label of $\mathbf{I}$ is given using the "winner takes all" principle by combing the scores of confidence calculated with all the segments of $\mathbf{I}$ :

$$
\operatorname{Label}(\mathbf{I}) \leftarrow \text { category } m^{*} ; \quad m^{*}=\underset{m=1,2, \ldots, M}{\arg \max }\left(\frac{1}{K_{o}} \sum_{i=1}^{K_{o}} \lambda_{m}\left(\mathbf{s}_{i}\right)\right)
$$

\section{NUMERICAL EXAMPLES AND DISCUSSIONS}

In this section, numerical examples on well-known benchmark image sets are presented to demonstrate the performance of the proposed approach.

\section{A. Experimental Setup}

In this paper, the following three aerial image sets are used for numerical examples. The example images of the three benchmark image sets are given in Fig. 3.

1) UCMerced dataset [7] 
UCMerced dataset consist of landuse images of 21 categories selected from aerial orthoimagery. This dataset has, in total, 2100 images of a size of $256 \times 256$ pixels. These images are uniformly labelled into the following 21 categories: i) agricultural; ii) airplane; iii) baseball diamond; iv) beach; v) buildings; vi) chaparral; vii) dense residential; viii) forest; $i x$ ) freeway; $x$ ) golf course; $x i$ ) harbour; xii) intersection; xiii) medium residential; $x i v)$ mobile home park; $x v$ ) overpass; $x v i)$ parking lot; xvii) river; xviii) runway; $x i x$ ) sparse residential; $x x$ ) storage tanks; and $x x i$ ) tennis courts. UCMerced dataset has a variety of categories, some of which are highly overlapping. Therefore, it is widely used as a benchmark.

\section{2) WHU-RS dataset [28]}

WHU-RS dataset is a popular benchmark dataset collected from Google Earth (Google Inc.). It consists of 950 images with a size of $600 \times 600$ pixels. This dataset has 19 categories, which include i) airport; ii) beach; iii) bridge; iv) commercial, v) desert; vi) farmland; vii) football field; viii) forest; $i x)$ industrial; $x$ ) meadow; $x i$ ) mountain; xii) park; xiii) parking lot; $x i v)$ pond; $x v$ ) port; $x v i$ ) railway; $x v i i)$ residential; xviii) river; and xix) viaduct, with 50 images in each. WHU-RS dataset contains aerial images with high variations in terms of illumination, scale resolution, etc., and, thus, is a difficult problem.

\section{3) RSSCN7 dataset [9]}

RSSCN7 dataset is collected from Google Earth (Google Inc.) as well. It has seven categories, and each one contains 400 images with the size of $400 \times 400$ pixels. The seven categories include: i) grassland; ii) forest; iii) farmland; iv) parking lot; v) resident; $v i)$ industry and vii) river and lake. The images of each category are sampled as four different scales (100 images per scale) with different angles, which make this problem a challenging one.

In this paper, we rescale the images of the WHU-RS dataset into the same size of the images of the RSSCN7 dataset, namely, $400 \times 400$ pixels, to avoid the loss of information during the segmentation operation.

For the training and validation sets separation, we follow the common practice by adopting two different sets [1], [3]. For UCMerced dataset, the ratios of the images for training per

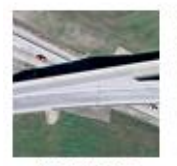

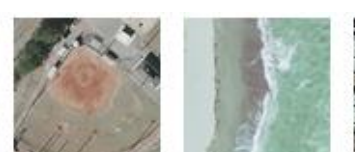

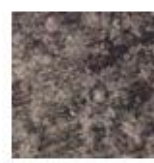

Forest

Overpass

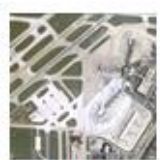

Airport

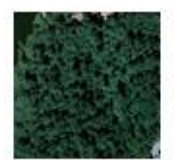

Forest
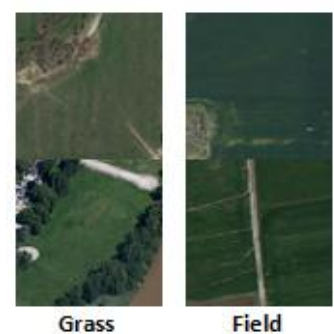
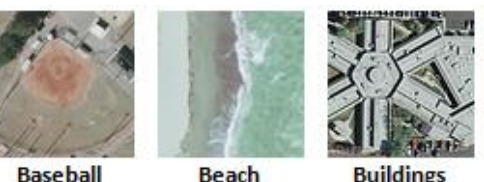

Diamond

Chaparral

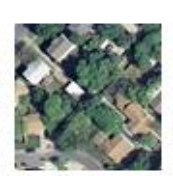

Medium

Residential

Golf Course

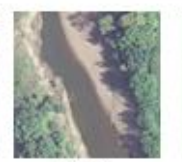

River

(a) UCMerced dataset
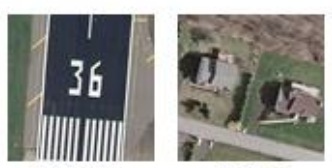

Sparse Residential
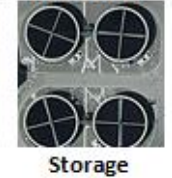

Tanks

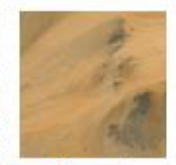

Desert
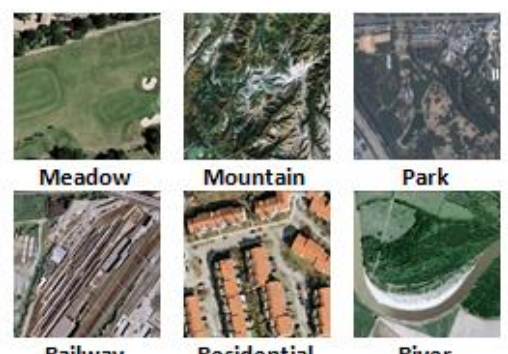

Railway Station

(b) WHU-RS dataset
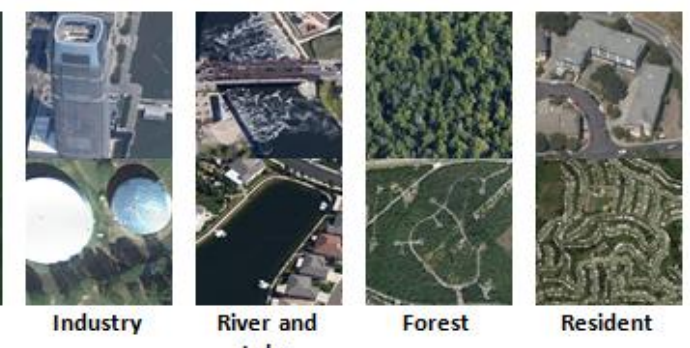

Resident

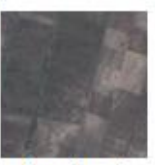

Farmland
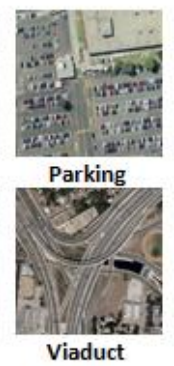

Lake

(c) RSSCN7 dataset

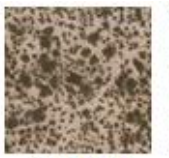

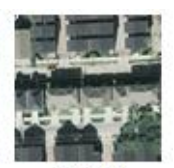

Dense

Residential

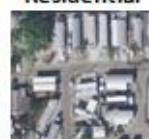

Mobile

Home Park

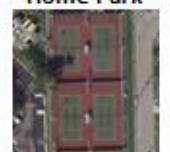

Tennis

Courts

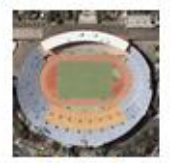

Football

Field

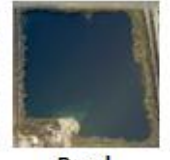

Pond
Fig. 3. Illustrative example of the three benchmark problesm

category are set to be $50 \%$ and $80 \%$. For WHU-RS dataset, the ratios are set to be $40 \%$ and $60 \%$. For RSSCN7 dataset, the ratios of the training images are set to be $20 \%$ and $50 \%$ per category.

The experimental results by the proposed approach are reported in the form of:

overall accuracy \pm standard deviation 
All the results are the average after 10 times Monte Carlo experiments. A variety of the state-of-the-art approaches are selected for comparison purposes.

\section{B. Experimental Results and Comparisons}

The experimental results on the UCMerced dataset obtained by the proposed approach as well as the selected state-of-the-art approaches are reported in Table I.

TABLE I. NUMERICAL RESUltS ON UCMERCED DATASET

\begin{tabular}{|l|c|c|}
\hline \multirow{2}{*}{\multicolumn{1}{|c|}{ Algorithm }} & \multicolumn{2}{c|}{$\begin{array}{c}\text { Percentage of Training Images } \\
\text { per Category }\end{array}$} \\
\cline { 2 - 3 } & $\mathbf{5 0 \%}$ & $\mathbf{8 0 \%}$ \\
\hline The proposed & $0.9402 \pm 0.0042$ & $0.9736 \pm 0.0086$ \\
\hline salM ${ }^{3}$ LBP-CLM [1] & $\mathbf{0 . 9 4 2 1} \pm \mathbf{0 . 0 0 7 5}$ & $0.9575 \pm 0.0080$ \\
\hline salM ${ }^{3}$ LBP [1] & $0.8997 \pm 0.0085$ & $0.9314 \pm 0.0100$ \\
\hline salCLM (eSIFT) [1] & $0.9293 \pm 0.0092$ & $0.9452 \pm 0.0079$ \\
\hline Combing Scenarios I and II [2] & & $\mathbf{0 . 9 8 4 9}$ \\
\hline Fine-tuning GoogleNet [29] & & 0.9710 \\
\hline CaffeNet [3] & $0.9398 \pm 0.0067$ & $0.9502 \pm 0.0081$ \\
\hline GoogLeNet [3] & $0.9270 \pm 0.0060$ & $0.9431 \pm 0.0089$ \\
\hline VGG-VD-16 [3] & $0.9414 \pm 0.0069$ & $0.9521 \pm 0.0120$ \\
\hline BoVW(SIFT) [3] & $0.7190 \pm 0.0079$ & $0.7412 \pm 0.0330$ \\
\hline VLAD(SIFT) [3] & $0.7323 \pm 0.0102$ & $0.7819 \pm 0.0166$ \\
\hline MS-CLBP+FV [30] & $0.8876 \pm 0.0079$ & $0.9300 \pm 0.0120$ \\
\hline
\end{tabular}

The experimental results obtained by the proposed approach on the WHU-RS dataset are reported in Table II. Comparison with the state-of-the-art is reported in the same table as well.

TABLE II. NUMERICAL RESUltS ON WHU-RS DATASET

\begin{tabular}{|c|c|c|}
\hline \multirow{2}{*}{ Algorithm } & \multicolumn{2}{|c|}{$\begin{array}{l}\text { Percentage of Training Images } \\
\text { per Category }\end{array}$} \\
\hline & $40 \%$ & $60 \%$ \\
\hline The proposed & $0.9579 \pm 0.0066$ & $0.9623 \pm 0.0070$ \\
\hline salM $^{3}$ LBP-CLM [1] & $0.9535 \pm 0.0076$ & $0.9638 \pm 0.0082$ \\
\hline salM ${ }^{3} L B P[1]$ & $0.8974 \pm 0.0184$ & $0.9258 \pm 0.0089$ \\
\hline salCLM (eSIFT) [1] & $0.9381 \pm 0.0091$ & $0.9592 \pm 0.0095$ \\
\hline Combing Scenarios I and II [2] & & 0.9889 \\
\hline CaffeNet [3] & $0.9511 \pm 0.0120$ & $0.9624 \pm 0.0056$ \\
\hline GoogLeNet [3] & $0.9312 \pm 0.0082$ & $0.9471 \pm 0.0133$ \\
\hline VGG-VD-16 [3] & $0.9544 \pm 0.0060$ & $0.9605 \pm 0.0091$ \\
\hline $\mathrm{BoVW}(\mathrm{SIFT})[3]$ & $0.7526 \pm 0.0139$ & $0.8013 \pm 0.0201$ \\
\hline VLAD(SIFT) [3] & $0.7637 \pm 0.0201$ & $0.8082 \pm 0.0215$ \\
\hline MS-CLBP+FV [30] & & $0.9453 \pm 0.0102$ \\
\hline
\end{tabular}

The experimental results obtained by the proposed approach on the RSSCN7 dataset are reported in Table III, and are compared with the state-of-the-art in the same table.

TABLE III. NUMERICAL RESUlTS ON RSSCN7 DATASET

\begin{tabular}{|l|c|c|}
\hline \multirow{2}{*}{ Algorithm } & \multicolumn{2}{c|}{$\begin{array}{c}\text { Percentage of Training Images } \\
\text { per Category }\end{array}$} \\
\cline { 2 - 3 } & $\mathbf{2 0 \%}$ & $\mathbf{5 0 \%}$ \\
\hline The proposed & $\mathbf{0 . 8 8 2 6} \pm \mathbf{0 . 0 0 5 6}$ & $\mathbf{0 . 9 1 7 5} \pm \mathbf{0 . 0 0 4 5}$ \\
\hline CaffeNet [3] & $0.8557 \pm 0.0095$ & $0.8885 \pm 0.0062$ \\
\hline GoogLeNet [3] & $0.8398 \pm 0.0087$ & $0.8718 \pm 0.0094$ \\
\hline VGG-VD-16 [3] & $0.8255 \pm 0.0111$ & $0.8584 \pm 0.0092$ \\
\hline BoVW(SIFT) [3] & $0.7633 \pm 0.0088$ & $0.8134 \pm 0.0055$ \\
\hline VLAD(SIFT) [3] & $0.7727 \pm 0.0058$ & $0.8082 \pm 0.0215$ \\
\hline DBNFS [9] & 0.7119 & 0.7581 \\
\hline
\end{tabular}

In Tables I, II and III, the best results reported are highlighted.

\section{Discussions}

Tables I, II and III demonstrate that the proposed approach is able to produce the state-of-the-art results in all three benchmark image sets. In particular, the proposed DRB approach outperforms all other comparative approaches on the RSSCN7 dataset. Therefore, one may conclude that the proposed approach is a strong alternative to other approaches for aerial image classification.

\section{CONCLUSION AND FUTURE WORK}

In this paper, a new deep rule-based (DRB) approach using an ensemble of high-level feature descriptors for aerial scene classification is proposed. With the discriminative semantic representations from the sub-regions of the aerial images extracted by the ensemble feature descriptor, the DRB approach is able to produce highly accurate classification results after a nonparametric, self-organizing and humaninterpretable learning process outperforming the alternatives. Numerical examples on benchmark datasets verify the proposed concept and principles.

As future work, we will investigate the performance of the DRB approach with different combinations of the high-level feature descriptors as well as different feature fusion strategies.

\section{REFERENCES}

[1] X. Bian, C. Chen, L. Tian, and Q. Du, "Fusing local and global features for high-resolution scene classification," IEEE J. Sel. Top. Appl. Earth Obs. Remote Sens., vol. 10, no. 6, pp. 2889-2901, 2017.

[2] F. Hu, G. S. Xia, J. Hu, and L. Zhang, "Transferring deep convolutional neural networks for the scene classification of high-resolution remote sensing imagery," Remote Sens., vol. 7, no. 11, pp. 14680-14707, 2015.

[3] G.-S. Xia, J. Hu, F. Hu, B. Shi, X. Bai, Y. Zhong, and L. Zhang, "AID: a benchmark dataset for performance evaluation of aerial scene classification," IEEE Trans. Geosci. Remote Sens., vol. 55, no. 7, pp. 3965-3981, 2017. 
[4] J. Yin, H. Li, and X. Jia, "Crater detection based on Gist features," IEEE J. Sel. Top. Appl. Earth Obs. Remote Sens., vol. 8, no. 1, pp. 23-29, 2015.

[5] G. Cheng, J. Han, P. Zhou, and L. Guo, "Scalable multi-class geospatial object detection in high-spatial-resolution remote sensing images," in International Geoscience and Remote Sensing Symposium (IGARSS), 2014, pp. 2479-2482.

[6] J. A. dos Santos, O. A. B. Penatti, and R. da Silva Torres, "Evaluating the potential of texture and color descriptors for remote sensing image retrieval and classification," in VISAPP, 2010, pp. 203-208.

[7] Y. Yang and S. Newsam, "Bag-of-visual-words and spatial extensions for land-use classification," in International Conference on Advances in Geographic Information Systems, 2010, pp. 270-279.

[8] S. Lazebnik, C. Schmid, and J. Ponce, "Beyond bags of features : spatial pyramid matching for recognizing natural scene categories," in IEEE Computer Society Conference on Computer Vision and Pattern Recognition, 2006, pp. 2169-2178.

[9] Q. Zou, L. Ni, T. Zhang, and Q. Wang, "Deep learning based feature selection for remote sensing scene classification," IEEE Geosci. Remote Sens. Lett., vol. 12, no. 11, pp. 2321-2325, 2015.

[10] G. J. Scott, M. R. England, W. A. Starms, R. A. Marcum, and C. H. Davis, "Training deep convolutional neural networks for land-cover classification of high-resolution imagery," IEEE Geosci. Remote Sens. Lett., vol. 14, no. 4, pp. 549-553, 2017.

[11] P. Angelov and X. Gu, Empirical approach to machine learning. Springer International Publishing, 2018.

[12] X. Gu, P. Angelov, C. Zhang, and P. Atkinson, "A massively parallel deep rule-based ensemble classifier for remote sensing scenes," IEEE Geosci. Remote Sens. Lett., vol. 15, no. 3, pp. 345-349, 2018.

[13] P. P. Angelov and X. Gu, "Deep rule-based classifier with human-level performance and characteristics," Inf. Sci. (Ny)., vol. 463-464, pp. 196213, 2018.

[14] A. Oliva and A. Torralba, "Modeling the shape of the scene: a holistic representation of the spatial envelope," Int. J. Comput. Vis., vol. 42, no. 3 , pp. 145-175, 2001.

[15] N. Dalal and B. Triggs, "Histograms of oriented gradients for human detection," in IEEE Computer Society Conference on Computer Vision and Pattern Recognition, 2005, pp. 886-893.

[16] Y. Jia, E. Shelhamer, J. Donahue, S. Karayev, J. Long, R. Girshick, S. Guadarrama, and T. Darrell, "Caffe: convolutional architecture for fast feature embedding*," in ACM International Conference on Multimedia, 2014, pp. 675-678.
[17] K. Simonyan and A. Zisserman, "Very deep convolutional networks for large-scale image recognition," in International Conference on Learning Representations, 2015, pp. 1-14.

[18] A. Krizhevsky, I. Sutskever, and G. E. Hinton, "ImageNet classification with deep convolutional neural networks," in Advances In Neural Information Processing Systems, 2012, pp. 1097-1105.

[19] M. L. Mekhalfi, F. Melgani, Y. Bazi, and N. Alajlan, "Land-use classification with compressive sensing multifeature fusion," IEEE Geosci. Remote Sens. Lett., vol. 12, no. 10, pp. 2155-2159, 2015.

[20] G. Sheng, W. Yang, T. Xu, and H. Sun, "High-resolution satellite scene classification using a sparse coding based multiple feature combination," Int. J. Remote Sens., vol. 33, no. 8, pp. 2395-2412, 2012.

[21] C. Szegedy, W. Liu, Y. Jia, P. Sermanet, S. Reed, D. Anguelov, D. Erhan, V. Vanhoucke, A. Rabinovich, C. Hill, and A. Arbor, "Going deeper with convolutions," in IEEE conference on computer vision and pattern recognition, 2015, pp. 1-9.

[22] K. He, X. Zhang, S. Ren, and J. Sun, "Deep residual learning for image recognition," in IEEE Conference on Computer Vision and Pattern Recognition (CVPR), 2016, pp. 770-778.

[23] B. Zhou, A. Lapedriza, J. Xiao, A. Torralba, and A. Oliva, "Learning deep features for scene recognition using places database," in Advances in neural information processing systems, 2014, pp. 487-495.

[24] P. Angelov and R. Yager, "A new type of simplified fuzzy rule-based system," Int. J. Gen. Syst., vol. 41, no. 2, pp. 163-185, 2011.

[25] https://uk.mathworks.com/matlabcentral/ leexchange/69012-empiricalapproach-to-machine-learning-software-package?s tid=prof contriblnk.

[26] P. Angelov and R. Yager, "Density-based averaging - a new operator for data fusion," Inf. Sci. (Ny)., vol. 222, pp. 163-174, 2013.

[27] P. P. Angelov, "Anomaly detection based on eccentricity analysis," in 2014 IEEE Symposium Series in Computational Intelligence, IEEE Symposium on Evolving and Autonomous Learning Systems, EALS, SSCI 2014, 2014, pp. 1-8.

[28] G. Xia, W. Yang, J. Delon, and Y. Gousseau, "Structural high-resolution satellite image indexing," in Proc. ISPRS TC 7th Symp. Years ISPRS, 2010, pp. 298-303.

[29] M. Castelluccio, G. Poggi, C. Sansone, and L. Verdoliva, "Land use classification in remote sensing images by convolutional neural networks," arXiv Prepr. arXiv 1508.00092, pp. 1-11, 2015.

[30] L. Huang, C. Chen, W. Li, and Q. Du, "Remote sensing image scene classification using multi-scale completed local binary patterns and fisher vectors," Remote Sens., vol. 8, no. 6, pp. 1-17, 2016. 